\title{
Fallopian Tube Catheterization
}

\author{
Amy Suzanne Thurmond, M.D. ${ }^{1}$
}

\section{ABSTRACT}

The delicate fallopian tube is the anatomic pathway to human reproduction. Simple, inexpensive tools and techniques allow radiologists to access this structure for promoting, and preventing pregnancy. Results from centers worldwide have shown that catheter recanalization is possible in $\sim 90 \%$ of women with proximal tubal occlusion using basic angiographic techniques familiar to all interventional radiologists. The American Society for Reproductive Medicine has recommended that patients in whom the hysterosalpingogram (HSG) demonstrates proximal tubal occlusion undergo fallopian tube catheterization with selective salpingography prior to more invasive and more costly infertility treatments. The pregnancy rate following fallopian tube recanalization is as high as $60 \%$ when the unblocked tubes are found to be normal and there is no history of tubal disease. In a population where the cause of infertility is less welldefined, tubal catheterization may not only help women conceive, but may also help to better define the underlying tubal abnormality. In the prevention of unwanted pregnancy, numerous materials and devices have been tested for blocking fallopian tubes. The ESSURE coil (Conceptus, Inc., Mountain View, CA) was FDA approved in 2002 for tubal sterilization by hysteroscopic placement. In some locales, radiologists are being asked to assist with this procedure or to place the coils fluoroscopically through fallopian tube catheterization.

KEYWORDS: Fallopian tube, hysterosalpingography, catheterization, fertility, sterilization

\begin{abstract}
Objectives: Upon completion of this article, the reader should be able to identify the technique, complications, and indications of fallopian tube catheterization by fluoroscopic guidance as well as explain the history and future directions that are presented.

Accreditation: Tufts University School of Medicine (TUSM) is accredited by the Accreditation Council for Continuing Medical Education to provide continuing medical education for physicians.

Credit: TUSM designates this educational activity for a maximum of 1 AMA PRA Category 1 Credit $^{\text {TM }}$. Physicians should only claim credit commensurate with the extent of their participation in the activity.
\end{abstract}

\section{FALLOPIAN TUBE ANATOMY}

The fallopian tube is a 7- to 9-cm, elongated trumpetshaped structure that extends from the cornu of the uterine cavity to the ovary. Its fimbriated end which is open to the peritoneal cavity, sweeps over the ovary, allowing the ovulated egg to be pulled into the fallopian tube where fertilization occurs. ${ }^{1}$ The proximal interstitial and isthmic portions closest to the uterus have a luminal diameter of $\sim 1 \mathrm{~mm}$, with a straight or slightly curved course in $\sim 60 \%$, and a tortuous or convoluted
${ }^{1}$ EPIC Imaging, Oregon Health Sciences University, Beaverton, Oregon.

Address for correspondence and reprint requests: Amy Suzanne Thurmond, M.D., Radiologist, EPIC Imaging, 8950 SW Nimbus Avenue, Beaverton, OR 97008 (e-mail: amyt@epicimaging.com).

Women's Issues in Interventional Radiology; Guest Editor,
Martha-Grace Knuttinen, M.D., Ph.D.

Semin Intervent Radiol 2008;25:425-431. Copyright (C) 2008 by Thieme Medical Publishers, Inc., 333 Seventh Avenue, New York, NY 10001, USA. Tel: +1(212) 584-4662.

DOI 10.1055/s-0028-1102995. ISSN 0739-9529. 
course in $40 \%$. This anatomy is thought to assist in preventing vaginal bacteria from gaining entrance to the body. At the same time, this tiny structure is prone to accumulation of secretions and scarring from inflammation, leading to unwanted sterility. ${ }^{2,3}$

\section{TRANSCERVICAL FALLOPIAN TUBE CATHETERIZATION}

In 1849, London, England, a surgeon probed a blocked fallopian tube with a whale bone in a woman with infertility. ${ }^{4}$ In the same year, a German physician cauterized the fallopian tube with silver nitrate to prevent pregnancy. ${ }^{5}$ Subsequent endeavors were limited by the materials and equipment available, which included rigid, then flexible hysteroscopes and catheterization techniques that evolved in the 1960s. Gynecologists were intimidated by an influential work published in 1961 that warned anyone from trying to probe the proximal tube because of its tortuousity. ${ }^{6}$ But by the mid-1980s, there were five separate reports describing transcervical catheterization of the fallopian tube using a coaxial angiographic technique. ${ }^{7-11} \mathrm{~A}$ series of seven women was reported at the Radiological Society of North America annual meeting in Chicago in $1987,{ }^{11}$ which resulted in numerous "hands-on" workshops and articles describing the technique, complications, technical success rates, and subsequent pregnancy rates. At the time, there was an intense interest around the world in advanced reproductive technologies for infertile women. In 1993, the American Society for Reproductive Medicine in their Guidelines for Practice recommended fallopian tube catheterization and recanalization as a first line procedure for diagnosis and treatment in women with proximal tubal occlusion. ${ }^{12}$

Meanwhile, investigators were exploring methods of blocking the fallopian tubes via transcervical fallopian tube catheterization. ${ }^{13-18}$ In 2002, the United States Food and Drug Administration (FDA) for the first

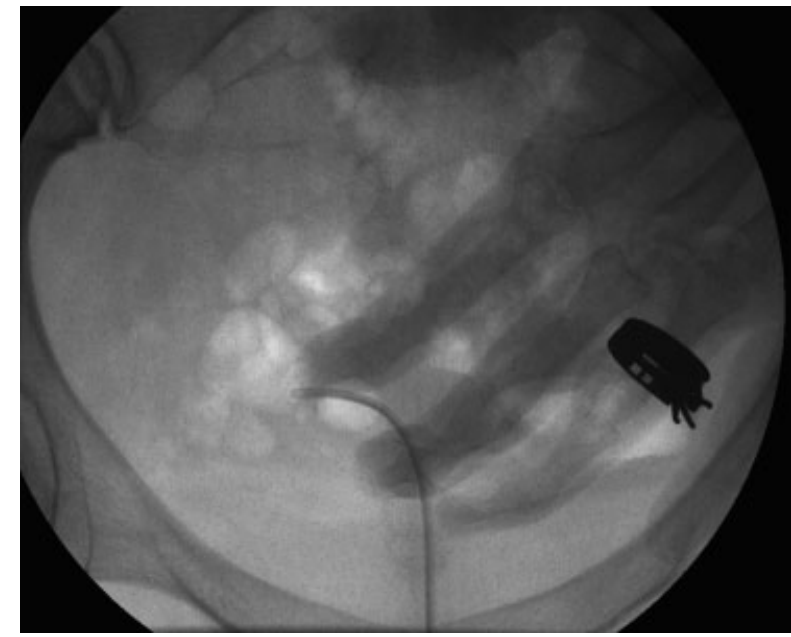

Figure 1 This woman is holding her pelvis in pain as the uterine catheter is inserted, a situation to be avoided. A calm demeanor and slow, steady movements allow these procedures to be done with no anesthesia or sedation.

time approved a method of transcervical sterilization. The ESSURE device, marketed by Conceptus Inc. (Mountain View, CA), is a small coil placed hysteroscopically to occlude the fallopian tubes. Animal testing of this device was done with fluoroscopic fallopian tube catheterization, identical to the technique used in women for unblocking the tubes. The company however, made a business decision to market the tubal occlusion device to gynecologists for placement with a hysteroscope.

\section{TECHNIQUE}

The procedure relies on a well-performed accurate hysterosalpingogram (HSG). Most women who have an HSG are apprehensive. The contrast agent should be injected slowly under fluoroscopic guidance to avoid sudden intense cramping of the uterus (Fig. 1). A condition traditionally called tubal spasm, but poorly
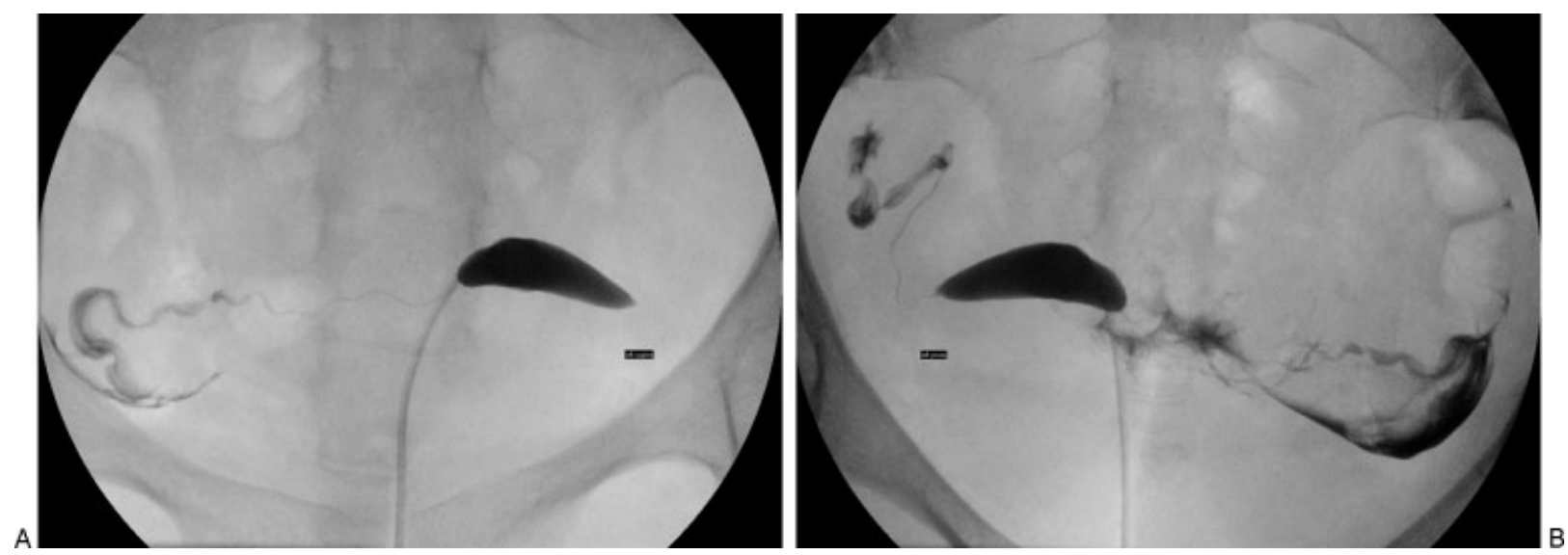

Figure 2 Apparent proximal tubal occlusion can occasionally be relieved by rolling the patient into a prone position. (A) Supine, the left tube appears occluded. (B) Prone, the left tube is patent. 

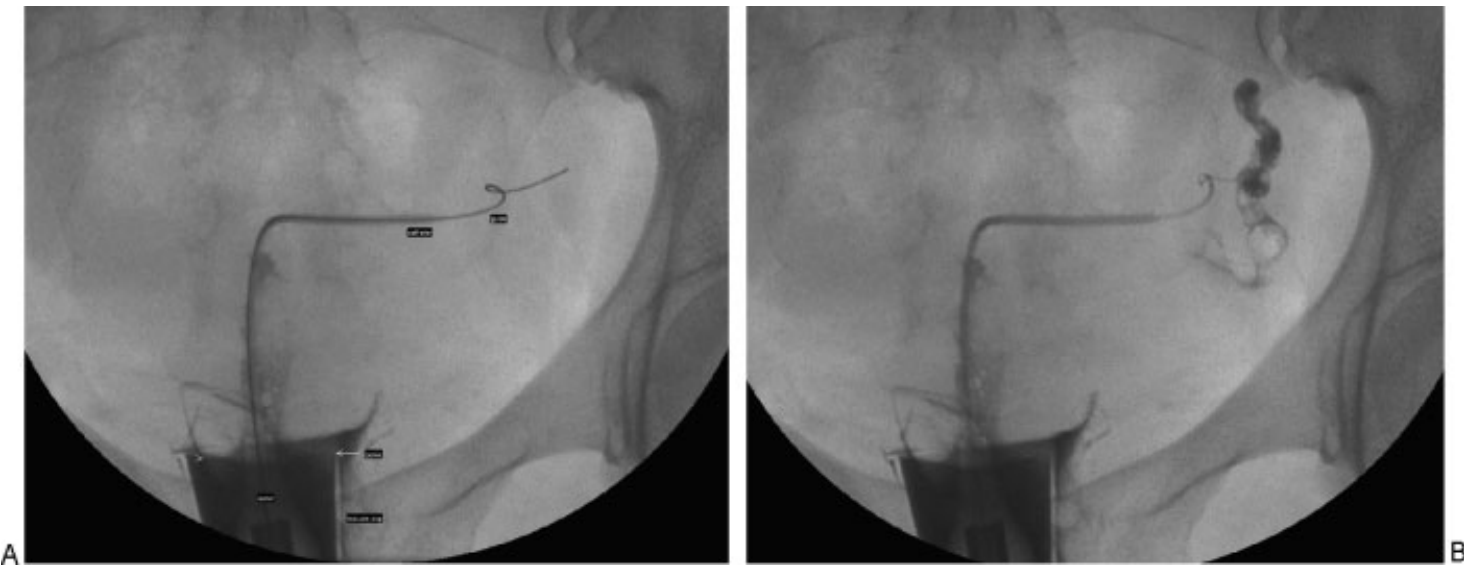

Figure 3 Basic technique of fallopian tube catheterization, recanalization, and selective salpingography. (A) Vacuum cup applied to the external cervix allows traction on the uterus without a tenaculum and coaxial introduction of a $5.5 \mathrm{Fr}$ catheter into the tubal ostium, followed by a $0.015^{\prime \prime}$ guidewire into the fallopian tube for dislodgement of debris. (B) Injection of contrast agent through a $3 \mathrm{Fr}$ catheter in the fallopian tube confirms successful recanalization and a normal-appearing patent tube.

understood, can result in temporary proximal tubal obstruction during the diagnostic test. ${ }^{19,20}$ If one or both tubes are not visualized, rolling the patient prone will sometimes result in tubal opacification (Fig. 2).

The equipment and techniques for fluoroscopic fallopian tube catheterization, selective salpingography, and recanalization are extensions of hyterosalpingography and angiography. There are two basic steps to the procedure: (1) uterine access, and (2) fallopian tube access. Uterine access requires a HSG device that promotes visualization of the uterine cavity and the tubal ostia, and allows traction to be placed on the cervix for subsequent catheterization. My preferred method consists of gaining access to the uterus with a vacuum cup HSG device (Fig. 3) (Thurmond-Rosch Hysterocath; Cook, Inc., Bloomington, IN or Cook, OB/Gyn, Spencer, IN). This provides a sterile conduit through which a series of coaxial catheters and guidewires can be introduced and allows traction on the uterus without the application of a tenaculum. A conventional HSG with diluted watersoluble contrast medium (Omnipaque $180 \mathrm{mgI} / \mathrm{mL}$ or something equivalent) is performed initially, which localizes the uterine cornua without obscuring the catheters.

For fallopian tube access, I prefer a coaxial catheter system, 9 Fr Teflon sheath and 5.5 Fr slightly curved polyethylene catheter advanced over a 0.035 -in diameter $(0.089 \mathrm{~cm}) \mathrm{J}$ guidewire to the uterine cornu (Cook, Inc., Bloomington, IN or Cook, OB/Gyn, Spencer, IN). This coaxial system of 3 devices is advantageous in that it allows the flexibility to catheterize the ostia in flexed or distorted uteri. The guidewire is removed and fullstrength contrast agent injected through the $5.5 \mathrm{Fr}$ catheter (selective salpingography).

If proximal tubal obstruction persists, a $0.015-$ in diameter $(0.038 \mathrm{~cm})$ guidewire with a flexible platinum tip and $3 \mathrm{Fr}$ Teflon catheter (Cook, Inc or Cook $\mathrm{OB} / \mathrm{Gyn}$ ) are advanced together into the fallopian tube and an attempt is made to recanalize the obstruction with gentle probing movements of the guidewire. If there is an acute angulation in the tube at the site of the obstruction, or if the obstruction is in the isthmic portion of the tube, a softer tapered guidewire and catheter are used (Tracker-18 and Taper guidewire, Target Therapeutics, Fremont, CA). The guidewire and catheter can be advanced as far as necessary to clear the obstruction, usually less than $4 \mathrm{~cm}$ from the tubal ostium. When the guidewire passes the obstruction, the guidewire is removed and contrast agent injected through the $3 \mathrm{Fr}$ catheter. Once the recanalization is completed, the $3 \mathrm{Fr}$ catheter is removed, and contrast agent is injected through the $5.5 \mathrm{Fr}$ catheter still wedged in the tubal ostium to better delineate the tube and visualize the site of

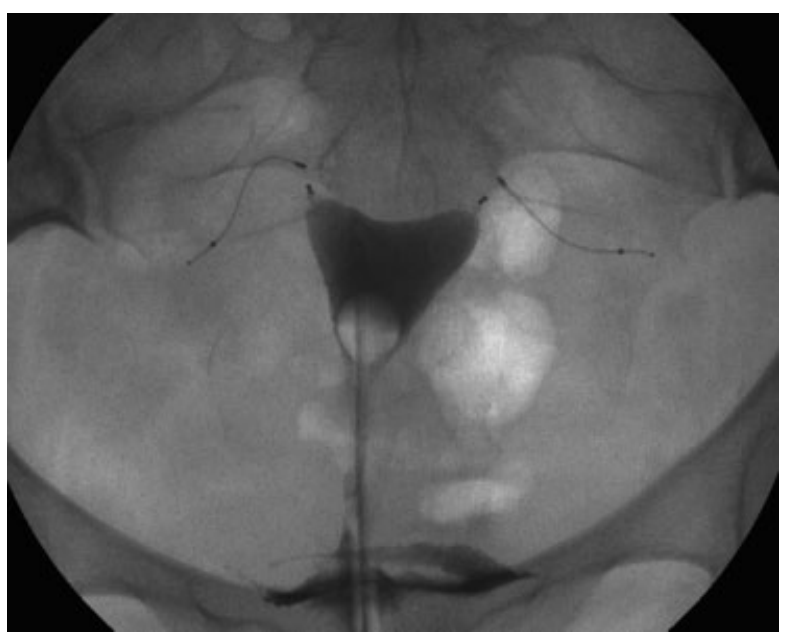

Figure 4 This hysterosalpingogram demonstrates ESSURE devices occluding both fallopian tubes for prevention of pregnancy, a procedure which can be performed by fluoroscopic fallopian tube catheterization. 
recanalization. A postrecanalization HSG can then be performed if desired.

The procedure is performed during the follicular phase of the menstrual cycle, utilizing sterile technique and with antibiotic prophylaxis (doxycycline $100 \mathrm{mg}$ orally twice daily for 5 days starting ideally 2 days before the procedure). A pregnancy test before the procedure is not necessary as long as the procedure is done in the follicular phase of the cycle, similar to the scheduling of a diagnostic HSG. Small doses of intravenous sedation and pain medication may be given, but are usually not necessary. No monitoring is required. It is not necessary to dilate the cervix or give paracervical anesthesia. The patient can usually be dismissed within 30 minutes of concluding the proce- dure. The patient can try to conceive the same week as the procedure.

The same technique can be used for placing the ESSURE device into the fallopian tube for tubal sterilization (Fig. 4) (RL Worthington-Kirsch, personal communication February 2008).

\section{COMPLICATIONS}

Mild uterine cramping and vaginal bleeding usually occur with fallopian tube catheterization. Intravenous sedation can be used, but is usually not necessary. Tubal perforation occurs $\sim 4 \%$ of the time and is usually related to the severity of underlying tubal disease. No additional monitoring or treatment is necessary when perforation
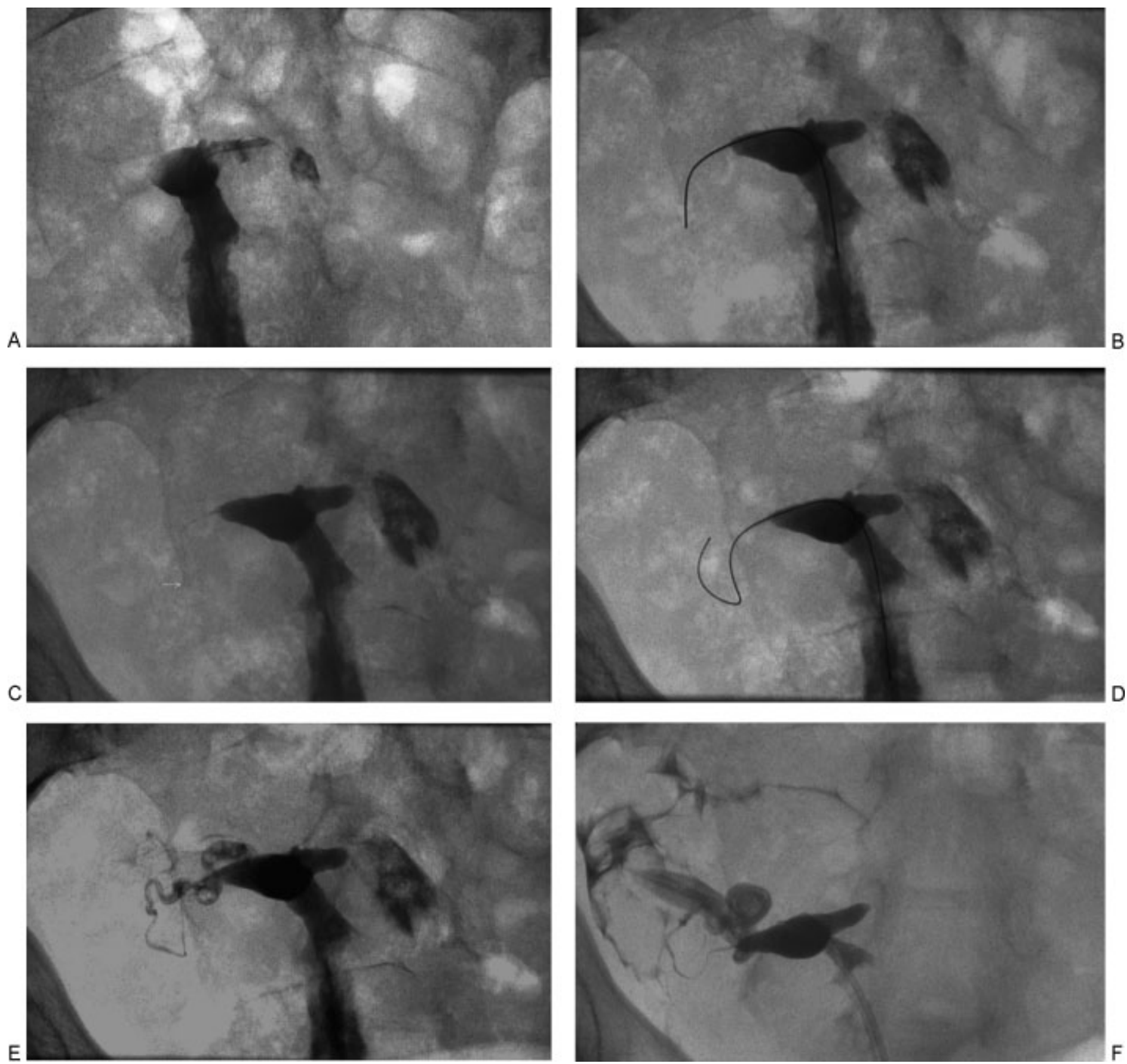

Figure 5 Attempt at bilateral tubal recanalization resulted in perforation of the left tube and patency of the right tube. (A) After passage of the guidewire on the left side, salpingogram demonstrates extravasation of contrast agent from a small tubal perforation. (B) Guidewire was then passed on the right side. (C) Salpingogram demonstrates persistent obstruction (arrow). (D) Guidewire was carefully passed again. (E) Right salpingogram demonstrates successful recanalization with a normalappearing patent tube. (F) Follow-up hysterosalpingogram 3 months later shows the left tube remains occluded and the perforation has healed, and the right fallopian tube remains patent status postcatheter-guidewire recanalization. 
occurs. Usually there is no reason to persist in recanalization attempts after the tube is perforated (Fig. 5).

The radiation dose to the ovaries during fluoroscopic catheterization has been documented to be less than $1 \mathrm{rad}(10 \mathrm{~Gy}) .^{21}$ This is in the same range as the radiation dose delivered during a barium enema or an intravenous pyelogram (IVP). The radiation dose varies depending on the equipment and the amount of fluoroscopy used as well as the number of radiographs exposed. A typical procedure takes less than 30 minutes and fluoro time is less than 10 minutes.

\section{RESULTS}

Many investigators agree that tubal recanalization is a treatment for infertility; however, this is difficult to prove because of the many clinical variables involved in achieving pregnancy. ${ }^{22}$ Particularly confusing, are the small number of patients who achieve pregnancy despite failed recanalization (ie, occluded tubes at the conclusion of the procedure). ${ }^{23}$

We prospectively evaluated the therapeutic effect of fallopian tube recanalization in 20 carefully selected patients in whom proximal tubal obstruction was thought to be the primary or sole cause of infertility. ${ }^{24}$ All patients had documented bilateral proximal tubal obstruction by at least two HSGs and by laparoscopy, with no distal tubal disease identified by laparoscopy. All 20 patients had been recommended for tubal microsurgery or in vitro fertilization, but underwent catheter recanalization instead. Recanalization of one or both tubes was successful in 19 women (95\%). By one year postprocedure, $58 \%$ of the women had conceived without receiving any other therapy, and all pregnancies were intrauterine. In a more heterogeneous group, including women with unilateral obstruction and peritubal adhesions, one may expect a lower short-term intrauterine pregnancy rate in the 20 to $40 \%$ range, and an $\sim 4 \%$ tubal pregnancy rate. ${ }^{24}$

The tubal reocclusion rate is difficult to determine because, in part, it is time dependent. In patients who do not conceive by 6 months, it appears that $\sim 50 \%$ of the tubes are reoccluded. ${ }^{24}$ If we assume that the tubes are patent in the patients who conceive, this gives an approximate reocclusion rate of $25 \%$. Repeat catheter recanalization is possible, and pregnancies have resulted after the second or even third procedure. ${ }^{24}$

Similar results for complications, tubal patency at the conclusion of the procedure, long-term patency, and pregnancy rates have been reported from around the world. $^{25-30}$

\section{CURRENT CONTROVERSIES AND FUTURE DIRECTIONS}

It is imperative to establish a close working relationship with the gynecologists and fertility specialists. Once the tubes are open, couples may need additional fertility treatments, which are out of a radiologist's field of expertise. More importantly, it is difficult to obtain referrals if the gynecologists do not know who you are or do not trust you. Establishing other services that benefit female patients, such as imaging-guided breast biopsies and uterine artery embolization can be helpful in developing a positive working relationship. Often the first women referred to you will be women with technically challenging anatomy or in whom the gynecologists have given up (Fig. 6). ${ }^{31-33}$ By helping the gynecologists in these difficult situations we "get our foot in the door" and establish our utility.
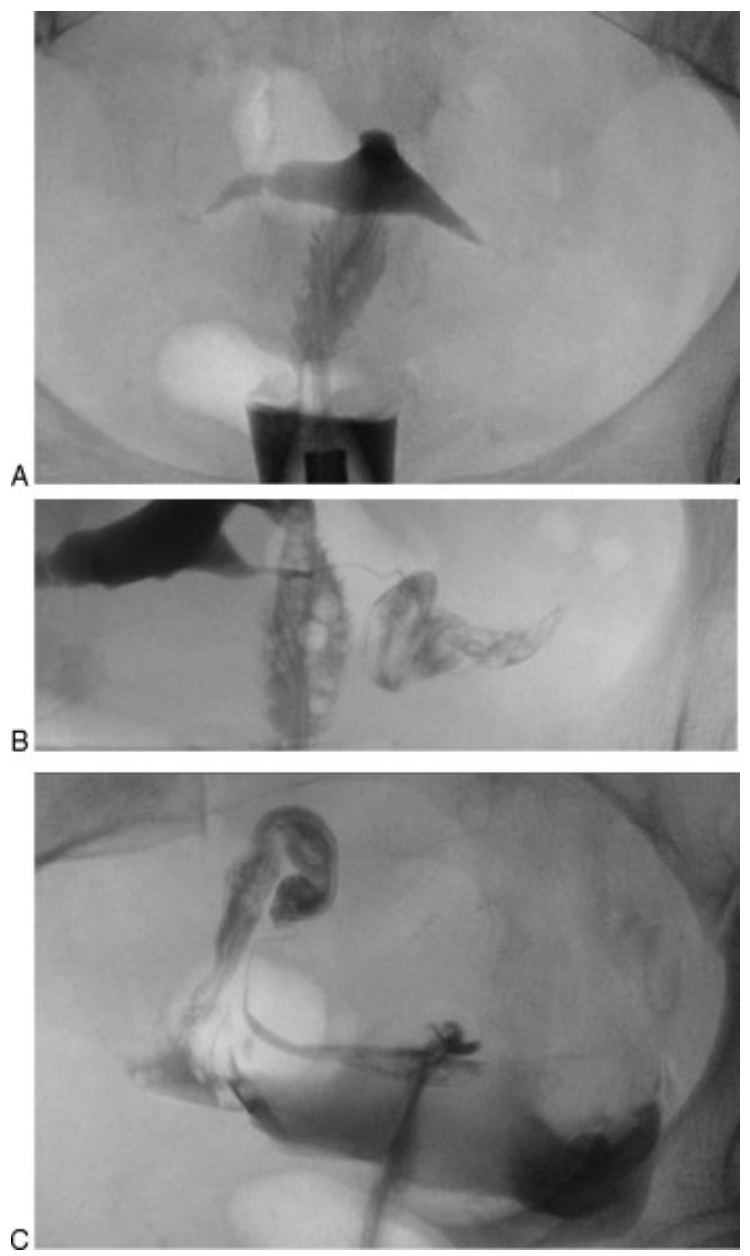

Figure 6 A 42-year-old woman with technically challenging anatomy and poor prognosis for pregnancy with failed ligation reversal surgery, followed by two cycles of in vitro fertilization that also failed. Catheter recanalization was successful, and she conceived the month following the procedure. (A) Hysterosalpingogram confirms occlusion of both tubes at the site of ligation reversal failure, and an anteflexed uterus. (B) Left tube was successfully recanalized; salpingogram demonstrates a somewhat shorter than normal tube consistent with the ligation reversal. (C) Right tube was also successfully recanalized and salpingogram demonstrates a widely patent tube. 
Fallopian tube catheterization for treatment of proximal tubal occlusion is by now well-established. However, the more recently trained fertility specialists are prone to treating most couples with some form of in vitro fertilization and embryo transfer (IVF) that bypasses the fallopian tubes altogether. This is because over the past 20 years the "take home baby rate" for IVF has gone from $5 \%$ to $75 \%$ in some clinics. ${ }^{34}$ IVF is still expensive and time consuming, and requires hormonal stimulation to which some couples object. It is our duty to remind the fertility specialists that catheter recanalization is a relatively inexpensive, minimally invasive treatment for proximal tubal occlusion. $^{35,36}$

In contrast to fallopian tube recanalization, transcervical occlusion of the fallopian tubes as a sterility procedure, is early in development. As of 2008, over 170,000 women worldwide have had ESSURE devices placed in their fallopian tubes by gynecologists to prevent pregnancies (US, Europe, Australia, New Zealand, Canada, Mexico, Central and South America). ${ }^{37} \mathrm{~A}$ handful of radiologists have been asked to place the device under fluoroscopic guidance when the hysteroscopic approach has failed (RL Worthington-Kirsch, personal communication, February 2008). Because fluoroscopic guidance is fast, easy, and inexpensive when performed by radiologists, this technique will likely gain momentum. ${ }^{38}$

\section{REFERENCES}

1. Woodruff JD, Pauerstein CJ, eds. The Fallopian Tube: Structure, Function, Management. Baltimore: Williams and Wilkins; 1969

2. Sulak PJ, Letterie GS, Coddington CC, et al. Histology of proximal tubal occlusion. Fertil Steril 1987;48:437-440

3. Papaioannou S. A hypothesis for the pathogenesis and natural history of proximal tubal blockage. Hum Reprod 2004;19:481-485

4. Smith WT. New method of treating sterility by removal of obstructions of the fallopian tubes. Lancet 1849;1:529-530

5. Friorep R. Zur Vorbeugung der Notwendigkeit des Kaiserschnitts und der perforation. Notiz Geburtshilfe Natur Heikunde 1849;221:9-10

6. Sweeney WJ. The interstitial portion of the uterine tube: its gross anatomy, course, and length. Obstet Gynecol 1962; $19: 3-8$

7. Rouanet JP, Chalut J. Une application du catheterisme selectif: la salpingographie. Nouv Presse Med 1977;31: 2785

8. Platia MP, Krudy AG. Transvaginal fluoroscopic recanalization of a proximally occluded oviduct. Fertil Steril 1985;44: 704-706

9. Confino E, Friberg J, Gleicher N. Transcervical balloon tuboplasty (TBT). Fertil Steril 1986;46:963-966

10. Jansen RPS, Anderson JC. Catheterization of the fallopian tube from the vagina. Lancet 1987;2:309-310
11. Thurmond AS, Novy M, Uchida BT, et al. Fallopian tube obstruction: selective salpingography and recanalization. Work in Progress. Radiology 1987;163:511-514

12. American Fertility Society. Guideline for Tubal Disease. Birmingham, AL: American Society for Reproductive Medicine (formerly American Fertility Society); 1993

13. Thurmond AS, Rosch J, Ross PL, et al. Transvaginal fallopian tube catheterization in an animal model. Invest Radiol 1988;23:818-821

14. Ross PL, Thurmond AS, Jones MJ, et al. Transcatheter tubal sterilization in rabbits: techniques and results. Invest Radiol 1994;29:570-573

15. Maubon AJ, Thurmond AS, Laurent A, et al. Selective tubal sterilization in rabbits: experience with a hydrogel combined with a sclerosing agent. Radiology 1994;193: 721-723

16. Schmitz-Rode T, Ross PL, Timmermans H, et al. Experimental nonsurgical female sterilization: trancervical implantation of microspindles in fallopian tubes. J Vasc Interv Radiol 1994;5:905-910

17. Maubon AJ, Thurmond AS, Laurent A, et al. Selective tubal sterilization in rabbits: experience with a hydrogel combined with a sclerosing agent. Radiology 1994;193:721-723

18. Maubon AJ, Thurmond AS, Nikolchev J, et al. Endoscopic transvaginal approach to the rabbit uterine cervix: improvement in the technique of selective tubal catheterization. Acad Radiol 1997;4:270-274

19. World Health Organization. Comparative trial of tubal insufflation, hysterosalpingography and laparoscopy with dye hydrotubation for assessment of tubal patency. Fertil Steril 1986;46:1101-1107

20. Thurmond AS, Novy M, Rosch J. Terbutaline in diagnosis of interstitial fallopian tube obstruction. Invest Radiol 1988;23:209-210

21. Hedgpeth PL, Thurmond AS, Fry R, et al. Radiographic fallopian tube recanalization: absorbed ovarian radiation dose. Radiology 1991;180:121-122

22. Thurmond AS. Interpretation of pregnancies after selective salpingography. Radiology 1994;190:11-13

23. Ferraiolo A, Ferraro F, Remorgida V, et al. Unexpected pregnancies after tubal recanalization failure with selective catheterization. Fertil Steril 1995;63:299-302

24. Thurmond AS, Rosch J. Nonsurgical fallopian tube recanalization for treatment of infertility. Radiology 1990;174: $572-573$

25. Kelekis D, Papageorgiou G, Fezoulidis I, et al. Selective transcervical recanalization of fallopian tubes: a method for diagnosis and treatment of infertility. J Interv Radiol 1992; 7:37-40

26. Maubon A, Rouanet JP, Cover S, et al. Fallopian tube recanalization by selective salpingography: an alternative to more invasive techniques? Hum Reprod 1992;7:14251428

27. Hayashi N, Kimoto T, Sakai T, et al. Selective fallopian tube catheterization: limited value in the treatment of fallopian tube diseases. Radiology 1994;190:141-143

28. Hovsepian DM, Bonn J, Eschelman DJ, et al. Fallopian tube recanalization in an unrestricted patient population. Radiology 1994;190:137-140

29. Thurmond AS, Machan LS, Maubon AJ, et al. A review of selective salpingography and fallopian tube catheterization. Radiographics 2000;20:1759-1768 
30. Papaioannou S, Afnan M, Sharif K. The role of selective salpingography and tubal catheterization in the management of the infertile couple. Curr Opin Obstet Gynecol 2004;16: 325-329

31. Thurmond AS, Jones MK, Mullick B, et al. Reversal of sterilization due to application of quinacrine by means of transcervical tubal catheterization. J Vasc Interv Radiol 1995; 6:147-149

32. Thurmond AS, Burry KA, Novy MJ. Salpingitis isthmica nodosa: results of transcervical fluoroscopic catheter recanalization. Fertil Steril 1995;63:715-722

33. Thurmond AS, Brandt KR, Gorrill MJ. Tubal obstruction after ligation reversal surgery: results of catheter recanalization. Radiology 1999;210:747-750

34. Society for Assisted Reproductive Technology of the American Fertility Society. Assisted reproductive technology in the United States: 1999 results generated from the American Society for Reproductive Medicine/Society for Assisted Reproductive Technology Registry. Fertil Steril 2002;78:918-931

35. Rawal N, Haddad N, Abbott GT. Selective salpingography and fallopian tube recanalisation: experience from a district general hospital. J Obstet Gynaecol 2005;25:586588

36. Das S, Nardo LG, Seif MW. Proximal tubal disease: the place for tubal cannulation. Reprod Biomed Online 2007; 15:383-388

37. Conceptus Inc. 2007 Annual Report and Form 10-K. Available at: www.conceptus.com. Accessed April 1, 2008

38. McSwain H, Brodie MF. Fallopian tube occlusion, an alternative to tubal ligation. Tech Vasc Interv Radiol 2006; 9:24-29 